\title{
Efectos de la fertilización del suelo sobre la producción de hojarasca en bosques pluviales tropicales del Chocó biogeográfico, Colombia
}

\author{
Effects of soil fertilization on litter production \\ in tropical rainforests of biogeographic Chocó, Colombia
}

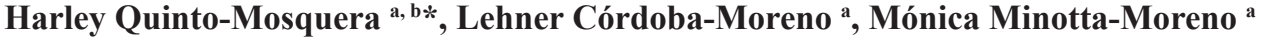 \\ a Universidad Tecnológica del Chocó “Diego Luis Córdoba”, Facultad de Ciencias Básicas, Programa de Biología, \\ Quibdó, Colombia, leancore1@hotmail.com, monicaslow@hotmail.com \\ * Autor de correspondencia: ${ }^{\mathbf{b}}$ Universidad Nacional de Colombia sede Medellín, Facultad de Ciencias Agrarias, \\ Doctorado en Ecología, Cl. 59a \#63-20, Medellín, Colombia, tel.: +57-3218154729, hquintom@unal.edu.co, \\ hquintom@gmail.com,d-harley.quinto@utch.edu.co
}

\begin{abstract}
SUMMARY
It has been hypothesized that the net primary productivity of low-altitude tropical rainforests is limited by the concentration of soil nutrients, mainly by edaphic phosphorus. To test this hypothesis, litter production was evaluated as a measure of net primary productivity in two tropical rain forests located in the towns of Opogodó and Pacurita in the biogeographic Chocó. One hundred and twenty-five leaf litter collectors were installed, which were monitored monthly for one year. These collectors were distributed in five fertilization treatments (control, nitrogen, phosphorus, potassium and NPK) to determine the effects of the addition of nutrients on the production of total litter and its components (leaves, branches, reproductive and miscellaneous material (material in decomposition)), and the reproduction / leaf ratio. In Opogodó, the application of nitrogen or phosphorus increased total litter production, nitrogen fertilization increased the production of branches, and the addition of nitrogen, phosphorus or potassium, separately, increased the production of miscellaneous material. Whereas, with fertilization, the production of reproductive material and the relation reproductive/leaves were reduced. In synthesis, fertilization changed the relative contribution of the different litter components, and favored the construction of photosynthetic structures (leaves), compared to reproductive structures. In addition, it was shown that the fall of litter is limited by nitrogen, phosphorus and potassium, which influence the carbon capture of these forest ecosystems. In Pacurita, fertilization had no significant effects on litter production.
\end{abstract}

Key words: phosphorus, nutritional limitation, nitrogen, potassium, net primary productivity.

\section{RESUMEN}

Se ha planteado la hipótesis de que la productividad primaria neta de bosques lluviosos tropicales de baja altitud se encuentra limitada por la concentración de nutrientes del suelo, principalmente por el fósforo edáfico. Para probar dicha hipótesis se evaluó la producción de hojarasca como una medida de la productividad primaria neta, en dos bosques pluviales tropicales ubicados en las localidades de Opogodó y Pacurita en el Chocó biogeográfico. Se instalaron 125 colectores de hojarasca, que se monitorearon mensualmente durante un año. Dichos colectores se distribuyeron en cinco tratamientos de fertilización (control, nitrógeno, fósforo, potasio y NPK), para determinar los efectos del adicionamiento de nutrientes sobre la producción de hojarasca total y sus componentes hojas, ramas, material reproductivo y miscelánea (material en descomposición), y la relación reproductivo/hojas. En Opogodó, la aplicación de nitrógeno o fósforo incrementó la producción de hojarasca total, la fertilización con nitrógeno aumentó la producción de ramas, y el adicionamiento de nitrógeno, fósforo o potasio, por separado, aumentó la producción de miscelánea. Mientras que, con la fertilización se redujo la producción de material reproductivo y la relación reproductivo/hojas. En síntesis, la fertilización cambió la contribución relativa de los diferentes componentes de la hojarasca, y favoreció la construcción de estructuras fotosintéticas (hojas), en comparación con las estructuras reproductivas. Además, se demostró que la caída de hojarasca está limitada de por nitrógeno, fósforo y potasio, que influyen sobre la captura de carbono de estos ecosistemas boscosos. En Pacurita, la fertilización no tuvo efectos significativos sobre la producción de hojarasca.

Palabras clave: fósforo, limitación nutricional, nitrógeno, potasio, productividad primaria neta.

\section{INTRODUCCIÓN}

La producción de hojarasca es uno de los principales componentes de la productividad primaria neta de bosques tropicales; puesto que representa $\approx 34 \%$ de la productividad primaria total de bosques en estado dinámico estable (Clark et al. 2001, Malhi et al. 2011). Dicha producción de hojarasca es fundamental para el funcionamiento de eco- 
sistemas boscosos porque constituye el principal mecanismo de flujo y reciclaje de nutrientes (Vitousek 1984, Vitousek y Sanford 1986); además, está regulada por factores biológicos y ambientales como la especie vegetal, edad y densidad del bosque, topografía, temperatura, precipitación y condiciones edáficas, entre otros (Bray y Gorham 1964, Chave et al. 2010). Siendo el contenido de nutrientes y el tipo de suelo los principales conductores de dicha producción en los trópicos (Vitousek 1984, Cleveland et al. 2011, Chave et al. 2010).

Vitousek (1984), luego de compilar información sobre la caída de hojarascay el reciclaje de nutrientes (nitrógeno, fósforo y calcio) de 62 bosques tropicales, observó que la producción de hojarasca se correlacionaba positivamente con el contenido foliar de fósforo. Con base en tales observaciones, se planteó la hipótesis de que la productividad primaria neta aérea se encuentra limitada principalmente por la disponibilidad de fósforo edáfico en bosques tropicales de baja altitud; mientras que, el nitrógeno del suelo por ser fijado biológicamente se le consideró como menos limitante en suelos tropicales antiguos (Vitousek 1984). Posiblemente, las altas tasas de meteorización del material parental que facilitan las pérdidas del fósforo edáfico por lixiviación, y además por la fijación del ion mineral en óxidos de hierro y aluminio que reducen su disponibilidad para las plantas, podrían ser consideradas como las principales causantes de tal limitación (Vitousek et al. 2010, Dalling et al. 2016). No obstante, dicha hipótesis aún no ha sido probada fehacientemente; pues, aunque algunos estudios han evidenciado correlaciones entre caída de hojarasca y disponibilidad edáfica y foliar de fósforo (Paoli y Curran 2007, Cleveland et al. 2011), biológicamente se entiende que una correlación no implica necesariamente relación de causalidad.

Para probar la hipótesis de la limitación nutricional del fósforo edáfico sobre la producción de hojarasca en bosques lluviosos tropicales de baja altitud se han realizado experimentos de fertilización con nitrógeno, fósforo y potasio (Sullivan et al. 2014, Sayer y Banin 2016). Sin embargo, tales experimentos han mostrado patrones de limitación contradictorios, pues algunos estudios reportan que la aplicación de nutrientes no afecta significativamente la producción de hojarasca (Cusack et al. 2011, AlvarezClare et al. 2013); mientras que otras investigaciones sí evidencian tal limitación (Mirmanto et al. 1999, Wright et al. 2011). Por ejemplo, Mirmanto et al. (1999) encontraron que con la adición de nutrientes de nitrógeno y fósforo aumentó significativamente la producción de hojarasca en bosques de tierras bajas en Indonesia. Mientras que Wright et al. (2011) reportaron un incrementó en la hojarasca con la aplicación de fósforo en bosques de Panamá. Asimismo, Sayer et al. (2012) observaron un incremento en la caída de hojarasca con la aplicación de nitrógeno, fósforo y materia orgánica en las épocas lluviosas del año. Por su parte, Kaspari et al. (2008) evidenciaron incrementos significativos en la producción del material reproductivo (flo- res, frutos y semillas) de la hojarasca con la aplicación de nitrógeno. Todo lo anterior evidencia una limitación de la producción de hojarasca, no solo por fósforo edáfico, sino por diversos nutrientes; así también, muestra que el efecto de la fertilización sobre la producción de hojarasca puede estar condicionado por la precipitación y diferir entre zonas con distinto nivel de lluvias, e incluso dentro de la misma zona según la época de mayor o menor lluvia, contrario a la hipótesis de Vitousek (1984). Además, muestra que la fertilización posiblemente afecta la asignación relativa de carbono en los componentes de la hojarasca.

A pesar de que la hipótesis de la limitación nutricional de la productividad aún está en debate, y que algunos experimentos de fertilización evidencian una limitación múltiple en los trópicos (Mirmanto et al. 1999, Sayer et al. 2012), estos análisis presentan una restricción adicional, pues todos los estudios de adición de nutrientes al suelo han sido realizados en zonas que cubren estrechos rangos de precipitación (entre 2.000 y $4.000 \mathrm{~mm}$ anuales) (Sullivan et al. 2014, Sayer y Banin 2016). Por lo tanto, se ignora qué efectos tiene la fertilización del suelo con nitrógeno, fósforo y potasio sobre la caída de hojarasca en bosques tropicales con alta pluviosidad ( $>5.000 \mathrm{~mm}$ anuales). Para responder a este interrogante se seleccionaron bosques tropicales del Pacífico colombiano, que poseen alta pluviosidad $(\approx 10.000 \mathrm{~mm}$ anuales), en los cuales se evaluaron los efectos de la fertilización edáfica sobre la caída total de hojarasca y sus componentes (hojas, ramas, flores, frutos, semillas y miscelánea). Asimismo, se evaluó el efecto de la aplicación de nutrientes sobre la contribución relativa de los distintos componentes de la hojarasca: reproductivos (flores, frutos y semillas) y fotosintéticos (hojas).

\section{MÉTODOS}

Área de estudio. El presente estudio se realizó en dos bosques pluviales tropicales ubicados en la región del Pacífico colombiano, uno en la localidad de Pacurita (municipio de Quibdó) y otro en la localidad de Opogodó (municipio de Condoto). Los suelos de ambos sitios son Ultisoles (Quinto y Moreno, 2016). Particularmente, la localidad de Opogodó, se encuentra ubicada a los 5'4'79" $\mathrm{N}-76^{\circ} 64^{\prime} 47^{\prime}$ ' W, con precipitación de $8.000 \mathrm{~mm}$ anuales, altitud de $70 \mathrm{~m}$ s.n.m, y topografía plana. Los suelos son arenosos (promedio \pm D.E. $85,7 \% \pm 6,57$ ), muy ácidos $(\mathrm{pH}=5,0 \pm 0,28)$, con baja capacidad de intercambio catiónico efectiva $\left(1,0 \pm 0,38 \mathrm{cmol}_{\mathrm{c}} \mathrm{kg}^{-1}\right)$ y contenidos muy bajos de fósforo $\left(1,32 \pm 0,60 \mathrm{mg} \mathrm{kg}^{-1}\right)$, magnesio $(0,28 \pm$ $\left.0,21 \mathrm{cmol}_{\mathrm{c}} \mathrm{kg}^{-1}\right)$ y calcio $\left(0,38 \pm 0,22 \mathrm{cmol}_{\mathrm{c}} \mathrm{kg}^{-1}\right)$. Las concentraciones de potasio son intermedias $\left(0,23 \pm 0,08 \mathrm{cmol}_{\mathrm{c}}\right.$ $\left.\mathrm{kg}^{-1}\right)$, y los valores de materia orgánica $(11,9 \pm 3,85 \%)$ y nitrógeno total $(0,61 \pm 0,22 \%)$ son altos. Las especies arbóreas dominantes son Wettinia quinaria (O.F. Cook et Doyle) Burret, Mabea occidentalis Benth, Calophyllum aurantiacum P.F. Stevens, y Oenocarpus bataua Mart (Quinto y Moreno 2016). 
La localidad de Pacurita, se encuentra ubicada a $5^{\circ} 41^{\prime} 55,8^{\prime \prime} \mathrm{N}-76^{\circ} 35^{\prime} 59,4^{\prime \prime} \mathrm{W}$ con precipitación de 10.000 $\mathrm{mm}$ anuales, altitud de 102-140 m s.n.m. y topografía quebrada. Los suelos son arcillosos y limosos, fuertemente ácidos ( $\mathrm{pH}=4,0 \pm 0,16)$, con porcentajes altos de saturación de aluminio $\left(57,2 \pm 9,61 \mathrm{cmol}_{\mathrm{c}} \mathrm{kg}^{-1}\right)$, y muy pobres de fósforo $\left(1,36 \pm 0,64 \mathrm{mg} \mathrm{kg}^{-1}\right)$, magnesio $(0,18 \pm 0,05$ cmol $\left.\mathrm{kg}^{-1}\right)$ y calcio $\left(0,35 \pm 0,10 \mathrm{cmol}_{\mathrm{c}} \mathrm{kg}^{-1}\right)$. Mientras que, la disponibilidad de potasio $\left(0,17 \pm 0,09 \mathrm{cmol}_{\mathrm{c}} \mathrm{kg}^{-1}\right)$ es intermedia, y la materia orgánica $(4,1 \pm 1,27 \%)$ y nitrógeno total $(0,20 \pm 0,06 \%)$ son altos. Los árboles y palmas dominantes son C. aurantiacum, O. bataua, Protium apiculatum Swart y Brosimum utile (Kunth) Pittier (Quinto y Moreno 2016).

En la localidad de Opogodó el muestreo se realizó en tres parcelas permanentes de una hectárea $(100 \times 100 \mathrm{~m})$, instaladas en un bosque ubicado en predios de la Universidad Tecnológica del Chocó "Diego Luis Córdoba". En la localidad de Pacurita el estudio se realizó en dos parcelas permanentes de las mismas dimensiones, ubicadas en una zona de reserva forestal denominada Estación Biológica Pacurita. En ambas localidades el muestreo se realizó en bosques primarios bien conservados.

Diseño experimental. Para evaluar el efecto de la fertilización del suelo sobre la Producción de hojarasca y sus componentes, se empleó un diseño en bloques completos aleatorizados con cinco tratamientos de fertilización (nitrógeno, fósforo, potasio, NPK y control) y cinco repeticiones. Para ello, cada parcela permanente se dividió en cinco unidades experimentales de $20 \times 100 \mathrm{~m}$ ( $0,2 \mathrm{ha})$, separadas por franjas de terreno de $2 \mathrm{~m}$ para reducir el riesgo de que los nutrientes migraran a otras parcelas por efectos de la escorrentía y/o topografía del suelo. En cada unidad experimental de $20 \times 100 \mathrm{~m}$ se aplicó aleatoriamente un tratamiento de fertilización. En Pacurita debido a la influencia de la topografía, la disposición espacial de las unidades experimentales fue paralela al gradiente de inclinación del terreno para reducir las pérdidas de nutrientes y la contaminación de las unidades vecinas. Cada unidad experimental de $20 \times 100 \mathrm{~m}$ se subdividió en cinco unidades de registro de $20 \times 20 \mathrm{~m}$, para facilitar la aplicación del fertilizante de modo más homogéneo y la posterior toma de datos de la producción de hojarasca.

Aplicación de tratamientos de fertilización. Los fertilizantes se aplicaron por el método de colocación "al voleo". Las dosis añadidas fueron similares a las reportadas en experimentos realizados en bosques lluviosos tropicales de baja altitud (Mirmanto et al. 1999, Wright et al. 2011). En las unidades de registro de $20 \times 20 \mathrm{~m}$, se aplicaron cuatro dosis iguales de fertilizantes a lo largo del año 2014, en los meses de febrero, mayo, agosto y noviembre, así: en el tratamiento de nitrógeno se aplicaron $125 \mathrm{~kg} \mathrm{ha}^{-1} \mathrm{año}^{-1}$ de nitrógeno en forma de urea $\left(\left(\mathrm{NH}_{2}\right)_{2} \mathrm{CO}\right)$, equivalente a $2,72 \mathrm{~kg}$ de urea por unidad de registro por aplicación; en el tratamiento de fósforo se adicionaron $50 \mathrm{~kg} \mathrm{ha}^{-1} \mathrm{año}^{-1} \mathrm{de}$ fósforo en forma de roca fosfórica $\left(\mathrm{H}_{3} \mathrm{PO}_{4}\right)$, equivalente a $1 \mathrm{~kg}$ de $\mathrm{H}_{3} \mathrm{PO}_{4}$ por unidad de registro por aplicación; en el tratamiento de potasio se aplicaron $50 \mathrm{~kg} \mathrm{ha}^{-1} \mathrm{año}^{-1} \mathrm{de}$ potasio en forma de cloruro de potasio $(\mathrm{KCl})$ ), equivalente a $1 \mathrm{~kg}$ de $\mathrm{KCl}$ por unidad de registro por aplicación; en el tratamiento de $\mathrm{N}+\mathrm{P}+\mathrm{K}$ se aplicaron conjuntamente cada una de las dosis mencionadas en los tratamientos anteriores; y en el control no se aplicaron fertilizantes.

Cuantificación de la producción de hojarasca y sus componentes. En el centro de cada una de las unidades de registro de 20 x $20 \mathrm{~m}$, se instaló un colector de hojarasca. En total se instalaron 125 colectores (75 en Opogodó y 50 en Pacurita) para el muestreo de hojarasca. Los colectores (construidos con tubos de PVC y anjeo), se fabricaron con un área de cobertura rectangular de $1 \times 0,5 \mathrm{~m}$ y se instalaron a $1 \mathrm{~m}$ sobre el suelo. El material acumulado (hojarasca) en los colectores se recogió en bolsas plásticas para evitar pérdidas de material vegetal. Posteriormente, el material colectado fue trasladado al laboratorio, donde fue secado a $70{ }^{\circ} \mathrm{C}$ en un horno de ventilación forzada (Acequilab Ltda $\left.{ }^{\circledR}\right)$ y se pesó mediante una balanza analítica de precisión $(0,001 \mathrm{~g})$. Luego de ello, la hojarasca se separó cuidadosamente en sus componentes hojas, ramas con diámetro $\leq 2 \mathrm{~cm}$, material reproductivo (flores, frutos y semillas) y misceláneos (material en descomposición) para estimar la contribución relativa de las distintas fracciones. Este procedimiento se realizó mensualmente durante un año. Los valores de producción se expresaron en mega gramos por hectárea por año $\left(\mathrm{Mg} \mathrm{ha} \mathrm{a}^{-1} \mathrm{año}^{-1}\right)$.

Análisis estadístico de los datos. Para evaluar el efecto de la fertilización del suelo y las localidades (Opogodó y Pacurita) sobre la producción de hojarasca total y por componentes, se utilizó un análisis de varianza (ANOVA) de doble vía, y se evaluó el efecto de cada una de las variables independientes (localidad y tratamientos), y su interacción. Las diferencias entre tratamientos y el control se evaluó con la prueba de rangos múltiples de Duncan; dado que en algunos casos no se cumplieron los supuestos (normalidad y homogeneidad de varianzas) para pruebas paramétricas, evaluados con los estadísticos de Bartlett, Hartley y Kurtosis. Asimismo, para evaluar el efecto de la fertilización del suelo sobre la contribución relativa de los distintos componentes de la producción de hojarasca: reproductivos (flores, frutos y semillas) y fotosintéticos (hojas), se determinó la contribución relativa de material reproductivo en función del material fotosintético, para ello se dividió la producción de material reproductivo sobre la producción de hojas (relación reproductivo/hojas) (Chave et al. 2010), y posteriormente se comparó su valor entre los distintos tratamientos de fertilización con las pruebas estadísticas mencionadas. Los análisis estadísticos se realizaron en el entorno de programación $\mathrm{R}$ (R Core Team 2012). 


\section{RESULTADOS}

En los bosques pluviales tropicales del Pacífico colombiano la producción de hojarasca total $\left(4,29 \mathrm{Mg} \mathrm{ha}^{-1}\right.$ año $\left.{ }^{-1}\right)$, hojas $\left(2,63 \mathrm{Mg} \mathrm{ha}^{-1}\right.$ año-1 $)$, ramas y tallos $(0,72 \mathrm{Mg}$ ha ${ }^{-1}$ año $\left.{ }^{-1}\right)$, y miscelánea $\left(0,72 \mathrm{Mg} \mathrm{ha}^{-1}\right.$ año $\left.{ }^{-1}\right)$ fue mayor en los tratamientos control de la localidad de Opogodó, en comparación con lo registrado en los bosques de Pacurita (cuadro 1). Igualmente, la producción de hojarasca (total, hojas, ramas y miscelánea) fue significativamente mayor en la localidad de Opogodó (cuadro 1).

Mientras que, a nivel de tratamientos de fertilización se observó que el efecto fue significativo tan solo en hojarasca total, ramas y miscelánea, pero no en material reproductivo y hojas (cuadro 1). Por su parte, a nivel de los efectos de la interacción (localidad $v$ s tratamientos), se observó que estas no presentaron efectos significativos para los diferentes componentes de la hojarasca (cuadro 1); por tal razón, se evaluó el efecto de los tratamientos de fertilización en cada localidad por separado. Particularmente, la producción de hojarasca total incrementó significativamente con la aplicación de nitrógeno o fósforo en la localidad de Opogodó respecto del control. Por su parte, la producción de ramas y tallos aumentó significativamente con la adición de nitrógeno en los bosques de Opogodó. Mientras que, la producción de miscelánea aumentó significativamente con la aplicación de nitrógeno, fósforo y potasio en los bosques de Opogodó. Contrario a lo anterior, la fertilización disminuyó la producción de material reproductivo y la relación de material reproductivo/hojas. En general, los bosques de Opogodó mostraron mayor sensibilidad a los tratamientos de fertilización, pues en Pacurita ningún tratamiento de fertilización afectó significativamente a la producción de hojarasca.

Cuadro 1. Producción de hojarasca (total y fracciones) en cinco tratamientos de fertilización del suelo en dos bosques pluviales del Chocó biogeográfico, Colombia. Donde los valores de producción corresponden a promedio \pm desviación estándar. Los asteriscos $(*)$ indican el efecto significativo en la producción de hojarasca por localidad, tratamiento, y su interacción. *: $P<0,05 ; * *: P<0,01 ; * * *$ : $P<0,0001$. Ns: no significativo.

Litter production (total and fractions) in five soil fertilization treatments in two rainforests of the biogeographic Chocó, Colombia. Where the production values correspond to Average \pm Standard deviation. Asterisks $(*)$ indicate the significant effect on litter production by location, treatment and their interaction. The asterisks $(*)$ indicates $P<0.05 ;(* *)$ indicates $P<0.01 ;(* *)$ indicates $P<0.0001$. Ns is not significant.

\begin{tabular}{|c|c|c|c|c|c|c|c|c|}
\hline \multirow{3}{*}{ Localidad } & \multicolumn{5}{|c|}{ Tratamiento } & \multicolumn{3}{|c|}{ Prueba F } \\
\hline & $\mathrm{N}$ & $\mathrm{P}$ & $\mathrm{K}$ & NPK & Control & Localidad & Tratamiento & Interacción \\
\hline & \multicolumn{8}{|c|}{ Producción de hojarasca total $\left(\mathrm{Mg} \mathrm{ha}^{-1}\right.$ año $\left.^{-1}\right)$} \\
\hline Pacurita & $3,29 \pm 1,13$ & $3,22 \pm 0,62$ & $2,97 \pm 0,68$ & $3,03 \pm 0,46$ & $3,15 \pm 0,57$ & \multirow{2}{*}{$90,8^{* * *}$} & \multirow{2}{*}{$2,64^{*}$} & \multirow{2}{*}{ Ns } \\
\hline \multirow[t]{2}{*}{ Opogodó } & $5,41 \pm 1,11$ & $5,07 \pm 0,93$ & $4,41 \pm 1,04$ & $4,98 \pm 1,33$ & $4,29 \pm 0,93$ & & & \\
\hline & \multicolumn{8}{|c|}{ Producción de hojas $\left(\mathrm{Mg} \mathrm{ha}^{-1}\right.$ año-1) } \\
\hline Pacurita & $2,20 \pm 1,08$ & $2,07 \pm 0,44$ & $1,98 \pm 0,54$ & $2,12 \pm 0,45$ & $2,19 \pm 0,49$ & \multirow{2}{*}{$51,34 * * *$} & \multirow{2}{*}{ Ns } & \multirow{2}{*}{ Ns } \\
\hline \multirow[t]{2}{*}{ Opogodó } & $3,13 \pm 0,73$ & $3,12 \pm 0,47$ & $2,62 \pm 0,72$ & $2,99 \pm 0,64$ & $2,63 \pm 0,65$ & & & \\
\hline & \multicolumn{8}{|c|}{ Producción de ramas y tallos $\left(\mathrm{Mg} \mathrm{ha}^{-1}\right.$ año $\left.^{-1}\right)$} \\
\hline Pacurita & $0,45 \pm 0,21$ & $0,37 \pm 0,17$ & $0,35 \pm 0,13$ & $0,37 \pm 0,15$ & $0,37 \pm 0,10$ & \multirow{2}{*}{$75,95 * * *$} & \multirow{2}{*}{$2,66^{*}$} & \multirow{2}{*}{ Ns } \\
\hline \multirow[t]{2}{*}{ Opogodó } & $1,07 \pm 0,39$ & $0,92 \pm 0,56$ & $0,74 \pm 0,28$ & $1,01 \pm 0,72$ & $0,72 \pm 0,38$ & & & \\
\hline & \multicolumn{8}{|c|}{ Producción de material reproductivo ( $\mathrm{Mg} \mathrm{ha}^{-1}$ año $\left.^{-1}\right)$} \\
\hline Pacurita & $0,09 \pm 0,07$ & $0,11 \pm 0,12$ & $0,11 \pm 0,11$ & $0,08 \pm 0,08$ & $0,09 \pm 0,07$ & \multirow{2}{*}{ Ns } & \multirow{2}{*}{ Ns } & \multirow{2}{*}{ ns } \\
\hline \multirow[t]{2}{*}{ Opogodó } & $0,08 \pm 0,07$ & $0,06 \pm 0,05$ & $0,06 \pm 0,12$ & $0,10 \pm 0,14$ & $0,18 \pm 0,19$ & & & \\
\hline & \multicolumn{8}{|c|}{ Producción de miscelánea $\left(\mathrm{Mg} \mathrm{ha}^{-1}\right.$ año $\left.^{-1}\right)$} \\
\hline Pacurita & $0,56 \pm 0,13$ & $0,66 \pm 0,23$ & $0,53 \pm 0,16$ & $0,46 \pm 0,13$ & $0,50 \pm 0,12$ & \multirow{2}{*}{$53,73 * * *$} & \multirow{2}{*}{$2,62^{*}$} & \multirow{2}{*}{ Ns } \\
\hline \multirow[t]{2}{*}{ Opogodó } & $1,14 \pm 0,51$ & $0,97 \pm 0,21$ & $0,99 \pm 0,35$ & $0,89 \pm 0,22$ & $0,76 \pm 0,19$ & & & \\
\hline & \multicolumn{8}{|c|}{ Relación reproductivo/hojas } \\
\hline Pacurita & $0,053 \pm 0,05$ & $0,062 \pm 0,075$ & $0,059 \pm 0,063$ & $0,045 \pm 0,053$ & $0,042 \pm 0,031$ & \multirow{2}{*}{$4,28^{*}$} & \multirow{2}{*}{ Ns } & \multirow{2}{*}{ Ns } \\
\hline Opogodó & $0,026 \pm 0,02$ & $0,018 \pm 0,013$ & $0,024 \pm 0,048$ & $0,03 \pm 0,032$ & $0,07 \pm 0,07$ & & & \\
\hline
\end{tabular}




\section{DISCUSIÓN}

La pregunta de investigación planteada en esta investigación fue: ¿Qué efectos tiene la fertilización del suelo con nitrógeno, fósforo y potasio sobre la caída de hojarasca en bosques tropicales con excesiva pluviosidad $(>5.000 \mathrm{~mm}$ anuales)? A base de los resultados, se evidenció que la aplicación de nitrógeno o fósforo incrementó la producción de hojarasca total; igualmente, la fertilización con nitrógeno aumentó la producción de ramas, y el adicionamiento de nitrógeno, fósforo o potasio aumentó la producción de miscelánea. En consecuencia, estos resultados evidencian una limitación nutricional de la producción de hojarasca y de sus componentes en bosques pluviales tropicales del Pacífico colombiano; no solo por la disponibilidad de fósforo edáfico, sino por diversos nutrientes (limitación por diferentes nutrientes), como nitrógeno y potasio; lo cual es similar a lo reportado por Mirmanto et al. (1999) y Sayer et al. (2012), quienes encontraron que con la aplicación de nitrógeno y fósforo aumentó significativamente la producción de hojarasca en bosques lluviosos tropicales de baja altitud. Por tal razón, la limitación por diferentes nutrientes registrada en estos bosques, contradice la hipótesis de Vitousek (1984) sobre la limitación nutricional de la productividad primaria neta aérea por el fósforo edáfico.

El hecho de que la producción de hojarasca de los bosques del Pacífico colombiano esté limitada por diferentes nutrientes probablemente se debe a los altos niveles de pluviosidad registrados en la zona (Quinto y Moreno 2016); los cuales, aumentan considerablemente las pérdidas edáficas de diversos minerales $\left(\mathrm{P} \mathrm{NH}_{4}^{+}\right.$y $\left.\mathrm{NO}_{3}^{-}\right)$por lixiviación (Santiago et al. 2005, Quinto y Moreno 2016), reducen las tasas de nitrificación, y disminuyen la mineralización neta de nitrógeno (Alvarez-Clare y Mack 2011); lo que en conjunto, posiblemente genera una limitación de la productividad primaria neta del bosque. Particularmente, la limitación de la caída de hojarasca generada por el fósforo del suelo, en particular, es razonable porque su disponibilidad edáfica es muy baja $\left(\mathrm{P}=1,32 \mathrm{mg} \mathrm{kg}^{-1}\right)$. Mientras que, la limitación por el nitrógeno del suelo al parecer es más compleja, porque su concentración edáfica total es relativamente alta ( $\mathrm{N}=0,61 \%)$ (Quinto y Moreno 2016).

Posiblemente, la razón que explica la limitación por nitrógeno edáfico está relacionada con el control que ejerce la concentración de fósforo y la humedad del suelo sobre la fijación biológica de nitrógeno atmosférico (Vitousek et al. 2010); puesto que, la disponibilidad edáfica de fósforo es muy baja en los bosques estudiados, y esta situación posiblemente estaría limitando el proceso de fijación de nitrógeno atmosférico, y podría estar propiciando una colimitación de la producción de hojarasca por nitrógeno y fósforo (Vitousek et al. 2010). Por otra parte, la aplicación de potasio al suelo no incrementó la producción de hojarasca total, seguramente porque este nutriente presenta concentraciones edáficas intermedias $\left(\mathrm{K}=0,23 \mathrm{cmol}_{\mathrm{c}} \mathrm{kg}^{-1}\right)$ (Quinto y Moreno 2016), que podrían mitigar su limita- ción nutricional. Además, la disponibilidad edáfica de este mineral se ve poco afectada por la alta precipitación (Santiago et al. 2005, Quinto y Moreno 2016), debido probablemente a que es muy soluble y sus pérdidas por lixiviación (120-250 $\mathrm{kg} \mathrm{ha}^{-1} \mathrm{año}^{-1}$ ), son compensadas por los ingresos al suelo producto del lavado desde el dosel del bosque (lixiviación foliar) en las lluvias, con valores de entre 13 y $220 \mathrm{~kg} \mathrm{ha}^{-1}$ año $^{-1}$ en bosques húmedos tropicales (Vitousek y Sanford 1986). Adicionalmente, el ión $\mathrm{K}^{+}$es liberado fácilmente al suelo desde los residuos orgánicos en descomposición; por lo cual, seguramente en estos bosques hay un flujo y/o suministro apropiado del nutriente.

En los bosques de la localidad de Pacurita, la aplicación de fertilizantes no tuvo efectos significativos sobre la producción de hojarasca (cuadro 1); posiblemente, esta falta de efectos se debe a las condiciones ambientales y edáficas de la zona, y/o por la cantidad o tipo de fertilizante adicionado. Explícitamente, los bosques de esta zona presentan las lluvias más intensas ( $\geq 12.000 \mathrm{~mm}$ anuales), suelos arcillosos más compactos y con topografía quebrada e inclinada (Quinto y Moreno 2016); lo que en conjunto, facilita la escorrentía superficial y lixiviación de los nutrientes adicionados, y dificultan evidenciar sus efectos sobre la producción de hojarasca y sus componentes. Igualmente, estos resultados muestran que la limitación nutricional depende de factores como textura del suelo, topografía y precipitación que afectan en conjunto la dinámica de los nutrientes en el ecosistema (Sullivan et al. 2014, Dalling et al. 2016, Sayer y Banin 2016). En consecuencia, es probable que para evidenciar efectos de la fertilización del suelo sobre la producción de la localidad de Pacurita, sea necesario adicionar una mayor cantidad de fertilizantes, y así compensar las pérdidas por lixiviación y escorrentía, o en efecto emplear otro tipo de fertilizantes, como inóculos de micorrizas, que seguramente mostrarían un mayor efecto. Otro factor que explica la poca influencia de la fertilización sobre la producción de hojarasca en los bosques de Pacurita es el hecho de que con la aplicación de minerales al suelo los tejidos vegetales lábiles (hojas) aumentan su contenido de nutrientes (Sayer y Banin 2016), y con dicho aumento se aceleran las tasas de descomposición de la hojarasca (Kaspari et al. 2008); por lo tanto, dado que en este estudio la hojarasca se muestreó mensualmente, es probable que un alto porcentaje se haya descompuesto antes de ser muestreada; más aún si se tiene en cuenta que, en bosques lluviosos tropicales entre el $30-70 \%$ de la hojarasca se descompone y mineraliza durante los primeros 30 días luego de que cae el material orgánico al suelo (Clark et al. 2001, Vendrami et al. 2012); lo cual, seguramente impidió evidenciar los efectos de la fertilización en esta localidad.

El hecho de que con la fertilización se incremente el contenido foliar de nutrientes (Sayer y Banin 2016), y que esto conlleve a un aumento en las tasas de descomposición de la hojarasca (Kaspari et al. 2008); podría seguramente explicar la alta producción de material en descomposición (miscelánea) registrado en los tratamientos de fertiliza- 
ción. Puesto que, una mayor disponibilidad de nutrientes ocasiona una mayor producción y descomposición de hojarasca (Mirmanto et al. 1999, Paoli y Curran 2007, Kaspari et al. 2008, Sayer y Banin 2016), lo que genera una mayor acumulación de material en descomposición (miscelánea) en el suelo. En consecuencia, la mayor miscelánea producida en Opogodó con la aplicación de nitrógeno, fósforo o potasio, corrobora la limitación que existe por estos minerales en los bosques pluviales del Chocó. Asimismo, el hecho de no evidenciar efectos de la fertilización sobre la producción de hojas; posiblemente se debe a que gran parte de las hojas caídas "ricas en nutrientes", pudieron haber entrado en un acelerado proceso de descomposición, dado sus altos contenidos de nutrientes (Sayer y Banin 2016) y el largo tiempo entre los muestreos (30 días); en consecuencia, es posible que este material orgánico haya sido muestreado indirectamente en el componente misceláneo de la hojarasca del bosque. Por lo tanto, es recomendable que para este tipo de ecosistemas lluviosos se recoja la hojarasca de los colectores con una mayor frecuencia, por ejemplo, cada 15 días, para evitar que se descomponga parte del material.

Se evidenció que la fertilización con nitrógeno favorece la producción de ramas en los bosques del Chocó, lo cual sugiere que los nutrientes adicionados podrían estar siendo empleados para construcción de estructuras destinadas para realizar fotosíntesis (renovación de hojas) y/o sus órganos de soporte (ramas); lo que corrobora la fuerte necesidad de adquisición de recursos como radiación solar y $\mathrm{CO}_{2}$ por parte de la planta. Estos resultados están relacionados con la hipótesis planteada por Bloom et al. (1985), quienes concluyeron que las plantas responden homeostáticamente al desequilibrio de recursos en el ambiente (por ejemplo competencia por luz y/o déficit de nutrientes), mediante la asignación de nueva biomasa vegetal para la adquisición de los recursos que son más limitantes del crecimiento (Bloom et al. 1985); en este caso la radiación solar. Asimismo, en estos ecosistemas se han evidenciado incrementos en producción y dinámica de raíces finas con la mayor disponibilidad y aplicación de nitrógeno y fósforo, como respuesta al déficit edáfico de nutrientes (Quinto 2016); lo cual, corrobora la hipótesis de Bloom et al. (1985), y evidencia el déficit al cual están sometidos estos bosques pluviales por radiación solar y nutrientes edáficos.

La aplicación de nutrientes no tuvo efectos significativos sobre la producción de material reproductivo en los bosques de Pacurita (cuadro 1); lo cual, es similar a lo reportado por DiManno y Ostertag (2016), quienes luego de aplicar nitrógeno y fósforo al suelo, no evidenciaron efectos sobre la producción de inflorescencias y semillas en bosques húmedos tropicales de Hawaii limitados por la disponibilidad de fósforo edáfico. Por su parte, en los bosques de Opogodó, con suelos arenosos y ricos en materia orgánica, la fertilización redujo marginalmente la caída de estructuras reproductivas; contrario a ello, Kaspari et al. (2008) evidenciaron un incremento en la producción de frutos y flores con la aplicación de nitrógeno en bosques con suelos relativamente fértiles de Panamá. Estos resultados en conjunto evidencian que, los efectos de la aplicación de nutrientes sobre la producción de estructuras reproductivas están determinados por la fertilidad del suelo. Al parecer en suelos fértiles la fertilización incrementa la producción de órganos reproductivos (Kaspari et al. 2008); mientras que, en suelos infértiles los efectos pueden ser nulos (DiManno y Ostertag 2016) e incluso negativos, como se evidenció en este estudio. Igualmente, estos resultados contradicen la hipótesis que plantea que en suelos pobres en nutrientes los árboles invierten menos en la producción de órganos reproductivos en relación con la producción de hojas (Chave et al. 2010). Puesto que, con la aplicación de fósforo y potasio se redujo la relación reproductivo/hojas, y se favoreció la producción de estructuras vegetativas (ramas) y fotosintéticas (hojas); lo cual, ratifica que la fertilización afecta a la contribución relativa de los distintos componentes de la hojarasca, favoreciendo la fotosíntesis, en detrimento de la producción de órganos reproductivos. Estos resultados están relacionados con lo expuesto anteriormente sobre la hipótesis de Bloom et al. (1985), en la cual se considera que las plantas asignan nueva biomasa (productividad primaria neta) para la adquisición de los recursos ambientales (luz y/o déficit de nutrientes) que son más limitantes del crecimiento; y dado que, con los tratamientos de fertilización se suplió el déficit de nutrientes, el recurso que podría empezar a ser más limitante es la radiación solar.

\section{CONCLUSIONES}

En el presente estudio se corrobora que la producción de hojarasca de los bosques pluviales tropicales del Pacífico colombiano se encuentra limitada por diferentes nutrientes (nitrógeno, fósforo y potasio). Igualmente, se evidencia que dicha limitación varía entre las localidades, debido a la influencia de factores como textura del suelo, topografía y precipitación. En la localidad de Opogodó, con topografía más plana y menor precipitación, la aplicación de nitrógeno o fósforo incrementa la producción de hojarasca total; además, la fertilización aumenta la producción de ramas y miscelánea, lo que muestra la influencia del nitrógeno, fósforo y potasio sobre la productividad primaria neta y funcionamiento de estos ecosistemas boscosos sometidos a alta pluviosidad. Mientras que, en la localidad de Pacurita, con topografías más quebradas y mayor precipitación, la aplicación de nutrientes no evidencia efectos significativos.

\section{AGRADECIMIENTOS}

Esta investigación fue financiada por el Convenio 0010-2013 firmado entre la Universidad Tecnológica del Chocó "Diego Luis Córdoba", la Universidad Nacional de Colombia Sede Medellín y el Departamento Administrati- 
vo de Ciencia, Tecnología e Innovación-COLCIENCIAS, en el marco del proyecto titulado: "Evaluación del efecto de la fertilización del suelo sobre la productividad primaria neta de bosques pluviales tropicales del departamento del Chocó (CÓDIGO: 1128-569-35113)". Esta investigación fue realizada, gracias al apoyo y hospitalidad de los habitantes de las localidades de Opogodó (municipio de Condoto) y Pacurita (municipio de Quibdó). Agradecemos los comentarios y sugerencias realizadas por el Doctor Flavio H. Moreno Hurtado, de la Universidad Nacional de Colombia sede Medellín.

\section{REFERENCIAS}

Alvarez-Clare S, MC Mack, M Brooks. 2013. A direct test of nitrogen and phosphorus limitation to net primary productivity in a lowland tropical wet forest. Ecology 94(7): 15401551. DOI: https://doi.org/10.1890/12-2128.1

Alvarez-Clare S, MC Mack. 2011. Influence of precipitation on soil and foliar nutrients across nine Costa Rican forests. Biotropica 43(4): 433-441. DOI: https://doi.org/10.1111/ j.1744-7429.2010.00732.x

Bloom AJ, FS Chapin III, HA Mooney. 1985. Resource limitation in plants - an economic analogy. Annual Review of Ecology and Systematics 16(1): 363-392. DOI: http://www. jstor.org/stable/2097053

Bray JR, E Gorham. 1964. Litter production in forests of the world. Advances in Ecological Research 2(1): 101-157. DOI: https://doi.org/10.1016/S0065-2504(08)60331-1

Chave J, D Navarrete, S Almeida, E Álvarez, LE Aragão, D Bonal, P Châtelet, JE Silva-Espejo, JY Goret, P von Hildebr, E Jiménez, S Patiño, MC Peñuela, OL Phillips, P Stevenson, Y Malhi. 2010. Regional and seasonal patterns of litterfall in tropical South America. Biogeosciences 7(1): 43-55. DOI: https://doi.org/10.5194/bg-7-43-2010

Clark DA, S Brown, DW Kicklighter, JD Chambers, JR Thomlinson, J Ni. 2001. Measuring net primary production in forest: Concepts and field methods. Ecological Aplications 11(2): 356-370. DOI: $\underline{10.2307 / 3060894}$

Cleveland CC, AR Townsend, P Taylor, S Alvarez-Clare, M Bustamante, G Chuyong, SZ Dobrowski, P Grierson, KE Harms, BZ Houlton, A Marklein, W Parton, S Porder, SC Reed, CA Sierra, WL Silver, EVJ Tanner, WR Wieder. 2011. Relationships among net primary productivity, nutrients and climate in tropical rain forest: a Pan-tropical analysis. Ecology Letter 14(9): 939-947. DOI: https://doi. org/10.1111/j.1461-0248.2011.01658.x

Cusack DF, WL Silver, MS Torn, WH McDowell. 2011. Effects of nitrogen additions on above and belowground carbon dynamics in two tropical forests. Biogeochemistry 104(1-3): 203-225. DOI: https://doi.org/10.1007/s10533-010-9496-4

Dalling JW, K Heineman, OR Lopez, SJ Wright, BL Turner. 2016. Nutrient availability in tropical rain forests: The paradigm of Phosphorus limitation. In G Goldstein, LS Santiago eds. Tropical tree physiology. Adaptations and responses in a changing environment. Zürich, Suiza. Springer International Publishing Switzerland. p. 261-273. DOI: 10.1007/9783-319-27422-5 12

DiManno NM, R Ostertag. 2016. Reproductive response to nitrogen and phosphorus fertilization along the Hawaiian archipelago's natural soil fertility gradient. Oecologia 180(1):245-255. DOI: 10.1007/s00442-015-3449-5

Kaspari M, MN Garcia, KE Harms, M Santana, SJ Wright, JB Yavitt. 2008. Multiple nutrients limit litterfall and decomposition in a tropical forest. Ecology Letters 11(1):35-43. DOI: $10.1111 / \mathrm{j} .1461-0248.2007 .01124 . \mathrm{x}$

Malhi Y, C Doughty, D Galbraith. 2011. The allocation of ecosystem net primary productivity in tropical forests. Philosophical Transactions of the Royal Society B: Biological Sciences 366 (1582): 3225-3245. DOI: 10.1098/rstb.2011.0062

Mirmanto E, J Proctor, J Green, L Nagy, Suriantata. 1999. Effects of nitrogen and phosphorus fertilization in a lowland evergreen rainforest. Philosophical Transactions of the Royal Society B 354(1391):1825-1829. DOI: $10.1098 /$ rstb.1999.0524

Paoli GD, LM Curran. 2007. Soil nutrients limit fine litter production and tree growth in mature lowland forest of southwestern Borneo. Ecosystems 10(3): 503-518. DOI: $10.1007 /$ $\underline{\mathrm{s} 10021-007-9042-\mathrm{y}}$

Quinto H. 2016. Evaluación del efecto de la fertilización del suelo sobre la productividad primaria neta de bosques pluviales tropicales del departamento del Chocó. Tesis de Doctorado en Ecología. Medellín. Colombia. Facultad de Ciencias Agrarias. Universidad Nacional de Colombia sede Medellín. $120 \mathrm{p}$.

Quinto H, FH Moreno. 2016. Precipitation effects on soil characteristics in tropical rain forests of the Chocó biogeographical region. Revista Facultad Nacional de Agronomía Medellin 69(1):7813-7823. DOI: http://dx.doi.org/10.15446/ rfna.v69n1.54749

Santiago LS, EAG Schuur, K Silvera. 2005. Nutrient cycling and plant-soil feedbacks along a precipitation gradient in lowland Panama. Journal of Tropical Ecology 21(4): 461-470. DOI: https://doi.org/10.1017/S0266467405002464

Sayer EJ, SJ Wright, EVJ Tanner, JB Yavitt, KE Harms, JS Powers, M Kaspari, MN Garcia, BL Turner. 2012. Variable Responses of Lowland Tropical Forest Nutrient Status to Fertilization and Litter Manipulation. Ecosystems 15(3): 387-400. DOI: https://doi.org/10.1007/s10021-011-9516-9

Sayer EJ, LF Banin. 2016. Tree nutrient status and nutrient cycling in tropical forest-Lessons from fertilization experiments. In G Goldstein, LS Santiago eds. Tropical tree physiology. Adaptations and responses in a changing environment. Zürich, Suiza. Springer International Publishing Switzerland. p. 275-297. DOI: https://dx.doi.org/10.1007/978-3319-27422-5 13

Sullivan BW, S Alvarez-Clare, SC Castle, S Porder, SC Reed, L Schreeg, CC Cleveland, AR Townsend. 2014. Assessing nutrient limitation in complex forested ecosystems: alternatives to large-scale fertilization experiments. Ecology 95(3): 668-681. DOI: $10.1890 / 13.0825 .1$

R Core Team. 2013. R: A language and environment for statistical computing. R Foundation for Statistical Computing, Vienna, Austria. Consulted 13 Aug. 2015. Available in http:// www.R-project.org/

Vendrami JL, C Jurinitz, C Castanho, L Lorenzo, A Oliveira. 2012. Litterfall and leaf decomposition in forest fragments under different successional phases on the Atlantic Plateau of the state of Sao Paulo, Brazil. Biota Neotropica 12(3): 134-141. DOI: $10.1590 /$ S1676-06032012000300016

Vitousek PM. 1984. Litterfall, nutrient cycling, and nutrient lim- 
itation in tropical forests. Ecology 65(1): 285-298. DOI: https://doi.org/10.2307/1939481

Vitousek PM, RL Sanford. 1986. Nutrient cycling in moist tropical forest. Annual Review of Ecology and Systematics 17(1):137-167. DOI: https://doi.org/10.1146/annurev. es.17.110186.001033

Vitousek P, S Porder, BZ Houlton, OA Chadwick. 2010. Terrestrial phosphorus limitation: mechanisms, implications, and nitrogen-phosphorus interactions. Ecological Applications 20(1):5-15. DOI: https://doi.org/10.1890/08-0127.1

Wright SJ, JB Yavitt, N Wurzburger, BL Turner, EVJ Tanner, EJ Sayer, LS Santiago, M Kaspari, LO Hedin, KE Harms, MN Garcia, MD Corre. 2011. Potassium, phosphorus, or nitrogen limit root allocation, tree growth, or litter production in a lowland tropical forest. Ecology 92(8): 1616-1625. DOI: https://doi.org/10.1890/10-1558.1

Recibido: $14 / 02 / 19$

Aceptado: 27/08/19 